\title{
PROPUESTA DE PROTOCOLO PARA EL SONDAJE DE DIAGNÓSTICO CLÍNICO EPIDEMIOLÓGICO PERIODONTAL
}

\section{PROPOSAL FOR A PROTOCOL FOR THE PERIODONTAL EPIDEMIOLOGICAL CLINICAL DIAGNOSIS PROBE}

\author{
Pinos-Ochoa Xavier. ${ }^{1 *}$ Pérez-Inga Ivan. ${ }^{1}$, Sisalima-Jara John ${ }^{1}$, Espinoza-Buestan Klever ${ }^{1}$, Niemes-Palacios \\ Gabriela ${ }^{1}$. \\ ${ }^{1}$ Odontólogo, Residente de la Especialidad de Periodoncia de la Universidad de Cuenca. Ecuador. \\ *xapo7@hotmail.com
}

\begin{abstract}
Resumen
En el presente artículo se propone un protocolo para el sondaje de diagnóstico clínico epidemiológico periodontal de tal manera de que los estudiantes e investigadores puedan obtener los datos periodontales rápida y eficazmente.
\end{abstract}

Palabras clave: Periodoncia, Periodontitis, Diagnóstico Clínico.

\begin{abstract}
This paper proposes a protocol for probing periodontal clinical epidemiological diagnosis in such a way that students and researchers can obtain periodontal data quickly and efficiently.
\end{abstract}

Key words: Periodontics, Periodontitis, Clinical Diagnosis.

\section{INTRODUCCIÓN}

El sondaje de diagnóstico epidemiológico periodontal es una herramienta que sirve para analizar o valorar el estado de salud periodontal de una población en un tiempo determinado. Esta propuesta pretende ser un instructivo de registro rápido de los datos periodontales obtenidos durante el diagnóstico epidemiológico clínico periodontal; pues hasta la fecha no existe literatura en español respecto a una secuencia rápida de obtención de datos como la que se propone en el presente artículo. De acuerdo al novedoso enfoque de la Odontología Basada en Evidencia OBE, los estudios epidemiológicos deben tener un método diagnóstico, económico y de rápida ejecución; en ese contexto proponemos este instructivo de registro rápido que facilite esta tarea y el propósito de este protocolo es ayudar a los estudiantes e investigadores a realizar una evaluación más rápida y eficiente.

\section{ESTADO DEL ARTE}

El correcto diagnóstico de la enfermedad periodontal es esencial para realizar una terapia periodontal exitosa que permite mejorar la calidad de vida de los pacientes y de la población en general. El sondaje periodontal complementa la evaluación visual inicial de la condición de los tejidos perio- dontales. La sonda periodontal es el instrumento diagnóstico más utilizado para la valorización clínica de la destrucción de los tejidos periodontales, ${ }^{1-3}$ que se compone de mango, cuello y punta de trabajo calibrada; éstas deben ser delgadas y el cuello angulado para permitir una fácil inserción al interior del surco o bolsa periodontal. (Figura 1). ${ }^{1,4,5}$

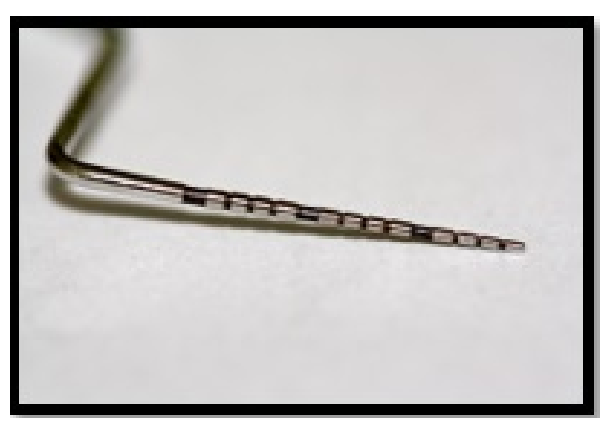

Fig. 1. Sonda Periodontal.

La sonda periodontal debe insertarse paralela al eje largo del diente, con una presión de 0.25 más menos $0.09 \mathrm{~N}$, (fuerza controlada incapaz de lesionar los tejidos periodontales. ${ }^{1,6}$ Se debe recorrer la periferia del diente en forma de saltos 
introduciendo y retirando la sonda, buscando las zonas con mayor profundidad de sondaje (Figura 8 y 9). Debido a que hay distintos diseños de sondas perio- dontales, se obtienen variaciones en los datos obtenidos. Actualmente la sonda que da más fiabilidad al procedimiento de diagnóstico, es la sonda Carolina del Norte. (Figura 1) $)^{1,7}$

Abreviaturas usadas:

- MG: Margen Gingival

- UCA: Unión Cemento Adamantina

- PS: Profundidad al Sondaje

- SS: Sangrado al Sondaje

- NIC: Nivel de Inserción Clínica

- LMG: Línea Mucogingival

- EA: Encía Adherida

- EQ: Encía Queratinizada

\section{TERMINOLOGÍA}

- Área de trabajo: es el espacio o lugar donde se coloca el instrumental odontológico (mesa odontológica).

- Campo operatorio: es el espacio o lugar donde se rea- liza el tratamiento odontológico (cavidad bucal y estructuras adyacentes).

- Profundidad de sondaje (PS): es la distancia en milímetros que marca la sonda periodontal que va desde el mar- gen gingival al fondo del surco o la bolsa periodontal. $1,4,8,9$

- Margen Gingival (MG): Es la distancia existente entre la UCA al borde de la encía libre. Cuando el MG está apical a la UCA se registra un número entero negativo. Cuando el MG está coronal a la UCA se registra un número entero positivo. ${ }^{1,4,5}$

- Nivel de Inserción Clínica (NIC): se define como la distancia desde la UCA al fondo del surco/ bolsa periodontal. $1,4,5$

- Sangrado al sondaje (SS): es un signo clínico que evidencia ulceración del epitelio del surco/bolsa periodontal, indicativo de actividad de la enfermedad periodontal. ${ }^{1,2,10}$

- Encía adherida (EA): sus límites son desde la línea de encía libre hasta LMG. ${ }^{1,4,5}$

- Encía queratinizada (EQ): sus límites son desde el borde de la encía libre hasta la $\mathrm{LMG}^{1,11}$

\section{CARACTERÍSTICAS DE LOS TEJIDOS GINGIVA- LES QUE SE DEBEN EVALUAR:}

- Color: pigmentaciones (Figura 2), enrojecimientos(Figura 3), lesiones blancas. ${ }^{1,12}$

- Forma: Contorno, arquitectura, festoneado en filo cuchillo(Figura 3$)^{1}$

- Consistencia: firme o edematosa (Figura 5) ${ }^{1,7}$

- Textura: punteado(Figura 4) / liso ${ }^{1}$

- Posición: recesión(Figura 6) / agrandamiento

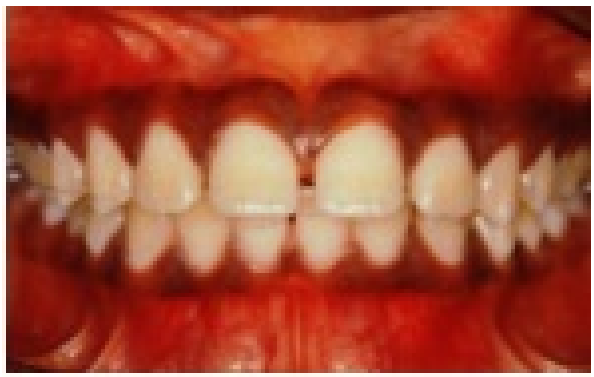

Fig. 2. Tejidos gingivales con pigmentaciones.

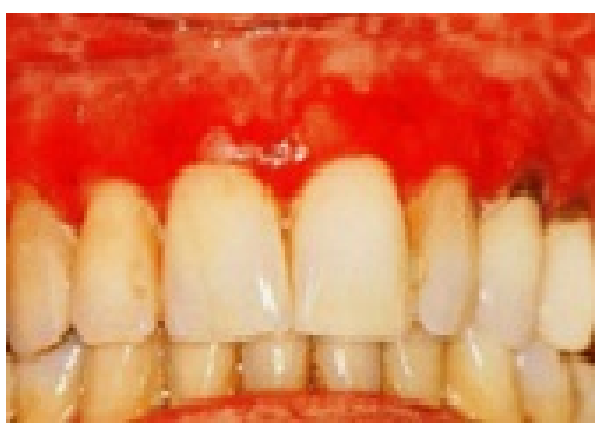

Fig. 3. Enrojecimiento de la encía.

\section{PROPUESTA}

El presente documento propone una nueva secuencia de pasos para el diagnóstico epidemiológico periodontal, desde la perspectiva de optimizar tiempo y simplificar procesos. De acuerdo a nuestra propuesta se debe realizar una inspección de los tejidos periodontales previa al sondaje periodontal, luego se procede a medir la profundidad de sondaje en tres puntos por superficie libre en todas las piezas presentes en la boca, a continuación se mide el margen gingival y posteriormente se establece el nivel de inserción clínica. Es importante tomar en cuenta la presencia de puntos de sangrado durante el sondaje, pues estos son evidencia de la actividad de la enfermedad periodontal. ${ }^{2,7}$ El correcto diagnóstico permitirá establecer el pronóstico y el plan de tratamiento específico para cada paciente.

\section{GENERALIDADES}

6.1. Materiales a utilizar.

A. lápiz, bolígrafo marcadores

- azul

- rojo

- verde

- negro

B. Ficha periodontal

6.2. Insumos Odontológicos: 


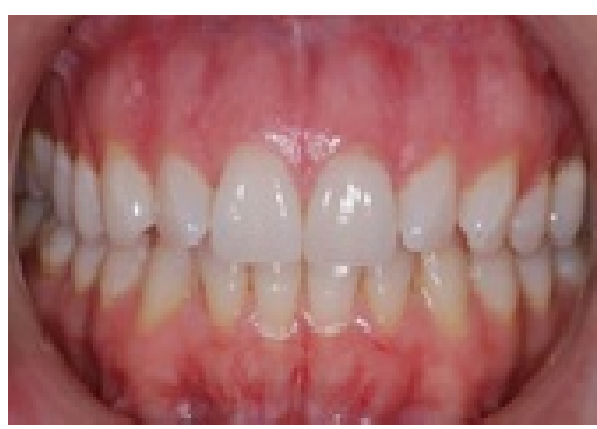

Fig. 4. Textura de la encía.

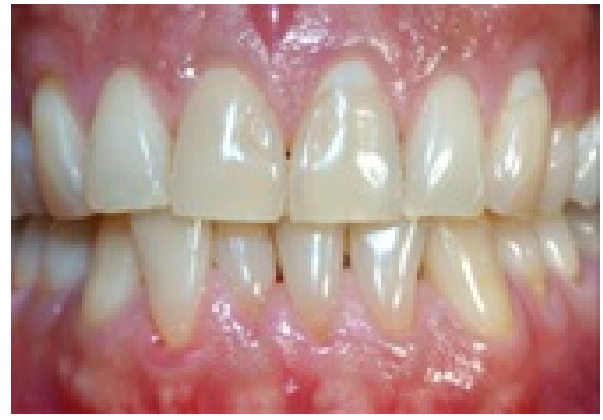

Fig. 5. Consistencia de la encía.

- Desinfectante en base a gluconato de clorhexidina $0.3 \%$ con cetrimida $3 \%$ dilución en agua $1 \mathrm{ml}$ : $100 \mathrm{ml}$ o alcohol metílico al $70 \%$.

- Gasas, guantes de examinación, mascarilla, eyector de saliva de plástico, gorro, gafas protectoras para paciente y operador, babero, uniforme odontológico anti fluido, pinzas porta babero.

\section{PROCEDIMIENTO}

\subsection{Normas de Bioseguridad}

- Desinfección del área de trabajo con gluconato de clorhexidina $0.3 \%$ con cetrimida $3 \%$ ) dilución en agua $1 \mathrm{ml}$ : $100 \mathrm{ml}$.

- Colocación de barrera protectora para la unidad dental que puede tener contacto con fluidos corporales.

- Colocación del instrumental estéril en el área de trabajo

- Recibir al paciente sin mascarilla ni guantes.

- Realización por parte del paciente de un enjuague del campo operatorio con $15 \mathrm{ml}$ de Clorhexidina al $0,12 \%$ por 1 min.

- Colocar al paciente en posición decúbito dorsal

- Lavado de manos con jabón antiséptico (yodopovidona al $10 \%$ ) por parte del operador y asistente.

- Colocarse gorro tanto el asistente como el operador

- Colocarse mascarilla tanto el asistente como el operador

- Colocarse los guantes tanto el asistente como el operador

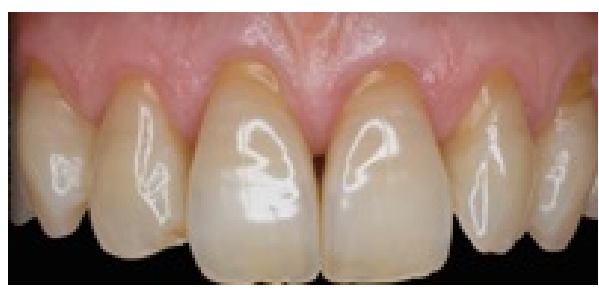

Fig. 6. Recisión gingival.

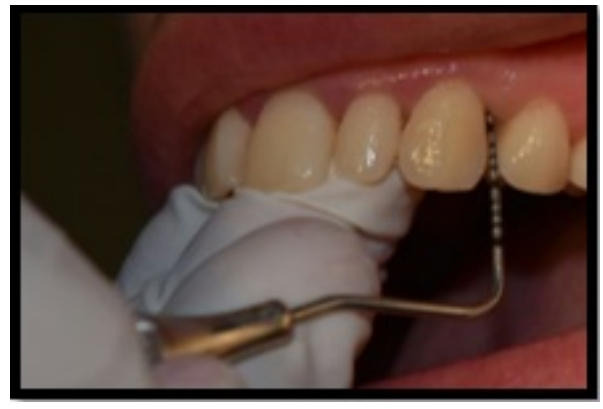

Fig. 7. Introducción de la sonda periodontal.

7.2 Examen Periodontal - Procedimientos clínicos de registros periodontales

- Antes de llamar al paciente el asistente debe realizar la recolección de datos de filiación y otros instrumentos de investigación (ej. cuestionario de autopercepción, calidad de vida, hábitos de higiene).

- El operador realizará la inspección visual y táctil de los tejidos periodontales (color consistencia, contorno, textura), y se registrarán estos datos.

- Registrar las piezas dentarias ausentes.

- Sondaje periodontal: Se introduce la sonda periodontal en un solo movimiento hasta el fondo del surco o bolsa periodontal (Figura 9), y con esto medimos la PS; luego retiramos ligeramente la sonda hasta localizar la UCA y desde aquí hasta el borde de la encía libre medimos el MG. El tercer valor (NIC) se obtiene con la diferencia de los datos anteriores. (Figura 9 y 10)

- La secuencia para el sondaje periodontal, se inicia por la cara bucal en distal de la última pieza presente, desde el cuadrante 1 hasta el cuadrante 2 . Se continúa el sondaje periodontal por la cara palatina, por distal de la última pieza presente desde el cuadrante 2 hasta el cuadrante 1 . A continuación realizamos el sondaje periodontal por la cara bucal en distal de la última pieza presente desde el cuadrante 4 hasta el cuadrante 3 . Se continúa el sondaje periodontal, por la cara lingual en distal de la última pieza presente desde el cuadrante 3 hasta el cuadrante 4.

- En cada diente se sondearán seis sitios, (mesial, medio y distal, tanto por la cara bucal como lingual/palatina). 


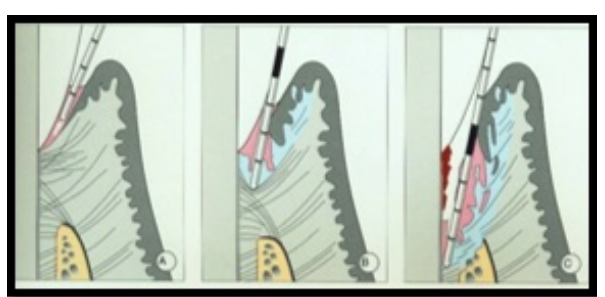

Fig. 8. Medición de la profundidad de sondaje en tres puntos.

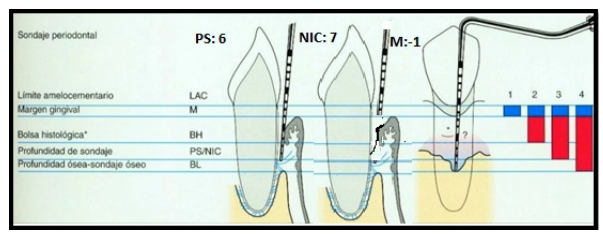

Fig. 9. Sonda periodontal insertada paralela al eje largo.

- El primer dato que se registre será la PS, el segundo valor registrado corresponderá al MG, y de la diferencia entre ambos valores se obtiene el NIC. (Figura 9 y 10).

- Una vez concluido el sondaje en cada cuadrante se confirma los datos registrados con la palabra CORRECTO.

- Se anota el sangrado al sondaje de cada sitio, si este se presenta una vez transcurridos 15 segundos después del sondaje.

- Siguiendo el mismo orden se registran los datos correspondientes a la encía adherida con la sonda medimos EQ desde el borde más apical del borde de la encía libre hasta la LMG y la diferencia de este valor con la PS obtenemos el valor de EA; exceptuando las caras palatinas y linguales.

- Terminado el proceso de sondaje, se le pide al paciente que realice un enjuague con agua corriente.

- Luego de la atención del paciente, quitarse los guantes mascarilla y gorro y desecharlos en el contenedor con bolsa roja.

- Lavarse las manos.

- Repetir la misma secuencia con el próximo paciente.

Conflicto de intereses y financiamiento Los autores declaran no tener conflicto de intereses, haber cumplido con los requisitos de autoría y haber autofinanciado este artículo.

\section{Referencias}

1 Armitage GC. Periodontal diagnoses and classification of periodontal diseases. Periodontol,2000, 2004; 34:9-21.

2 Joss A, Adler R, Lang NP. Bleeding on probing. A parameter for monitoring periodontal conditions in clinical practice. J Clin Periodontol,1994;21:402-408.20.

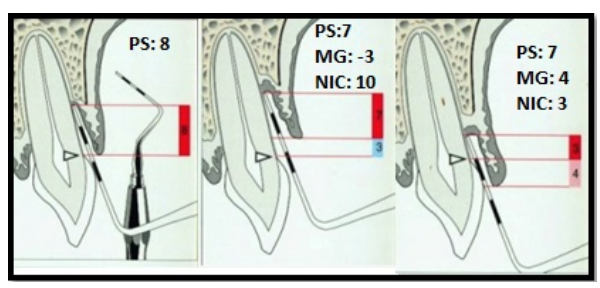

Fig. 10. localización de UCA.

3 Listgarten MA. Periodontal probing: what does it mean? J Clin Periodontol, 1980;7:165-176. 17.

4 Van der Velden U. Influence of periodontal health on probing depth and bleeding tendency. J. Clin Periodontol ,1980;7:129-139.24.

5 Rojas A. Informe de casos clínicos. Tesis digitales UNMSN

6 Hefti, A. (1997). Periodontal probing. Critical Reviews in Oral Biology \& Medicine, 8(3), pp.336-356.

7 Lang NP, Joss A, Orsanic T, Gusberti FA, Siegrist BE. Bleeding on probing. A predictor for the progression of periodiontal disease? J Clin Periodontol ,1986; 13:590-596.22.

8 Caton J, Greenstein G, Polson AM. Depth of periodontal probe penetrationrelated to clinical and histologic signs of gingival inflammation. J Periodontol ,1981;52:626-629.10.

9 Greenstein G. Current interpretations of periodontal probing evaluations: diagnostic and therapeutic implications. Compend Contin Educ Dent, 2005; 26:381-390.13.

10 Lang NP, Nyman S, Senn C, Joss A. Bleeding on probing as it relates to probing pressure and gingival health. J Clin Periodontol ,1991; 18:257-261.

11 Weinberg MA, Eskow RN. Periodontal terminology revisited. J Periodontol ,2003; 74: 563-565.9.

12 Diagnosis and classification of periodontal disease. Australian dental Journal 2009, 54: (1 Subpl):s11-s26.

Recibido: 10 de enero de 2017.

Aceptado: 17 de febrero de 2017. 